\title{
Eine Modifikation der Rieglerschen Methode, die Harnstoff- menge im Harn zu bestimmen.
}

\author{
Von \\ Th. Ekecrantz und K. A. Söderman. \\ Mit einer Abbildung im Text. \\ (Aus der chemischen Abteilung des Pharmazeutischen Instituts zu Stockholm.) \\ (Der Redaktion zugegangen am 1. November 1911.)
}

Von den für die Bestimmung des Harnstoffgehalts im Harn vorgeschlagenen Methoden ist die von $\mathrm{Riegler}^{1}$ ) angegebene, die hinreichend genaue Resultate liefert und nur wenig Zeit in Anspruch nimmt, ohne Zweifel die häufigst angewandte. Da diese Methode darauf basiert ist, daß Harnstoff beim Erwärmen mit Salpetersäure, die ein bißchen salpetrige Säure enthält, in gleiche Volumina Kohlenstoffdioxyd und Stickstoff $\left(\mathrm{CO}_{2}+\mathrm{N}_{2}\right)$ zersetzt wird, kann man, ohne daß die Bestimmung längere Zeit in Anspruch nimmt, durch Kombination des Rieglerschen Verfahrens und der Dumasschen Methode der Stickstoffbestimmung in organischen Verbindungen, in einer sehr einfachen Weise den Harnstoffgehalt im Harn bestimmen.

Der bei Ausführung der Bestimmung angewandte Apparat besteht aus folgenden Teilen: Das Meßrohr $d$, in $1 / 20 \mathrm{ccm}$ geteilt, hat nahe dem Boden zwei Seitenrohre, das eine mit der Niveaukugel $f$ (50\% ige Kalilauge enthaltend), das zweite mit dem Gasentwicklungsgefäß $a$ in Verbindung stehend. Durch Heben und Senken der Niveaukugel bei offenem Hahn $e$ kann man nach Wunsch das Meßrohr füllen oder entleeren. Das Gasentwicklungsgefäß $a$ ist mit einem Kautschukstopfen, der einen zylindrischen, graduierten Scheidetrichter und zwei Glas-

1) Zeitschrift für analyt. Chemie, Bd. 33, S. 49. - Apoth.-Zeitung 1897, S. 89. 
röhren enthält, geschlossen. Die erste von diesen Röhren ist nahe unter dem Stopfen abgeschnitten und dient als Ableitungsrohr für die Gasmischung, das zweite, $h$, geht etwa

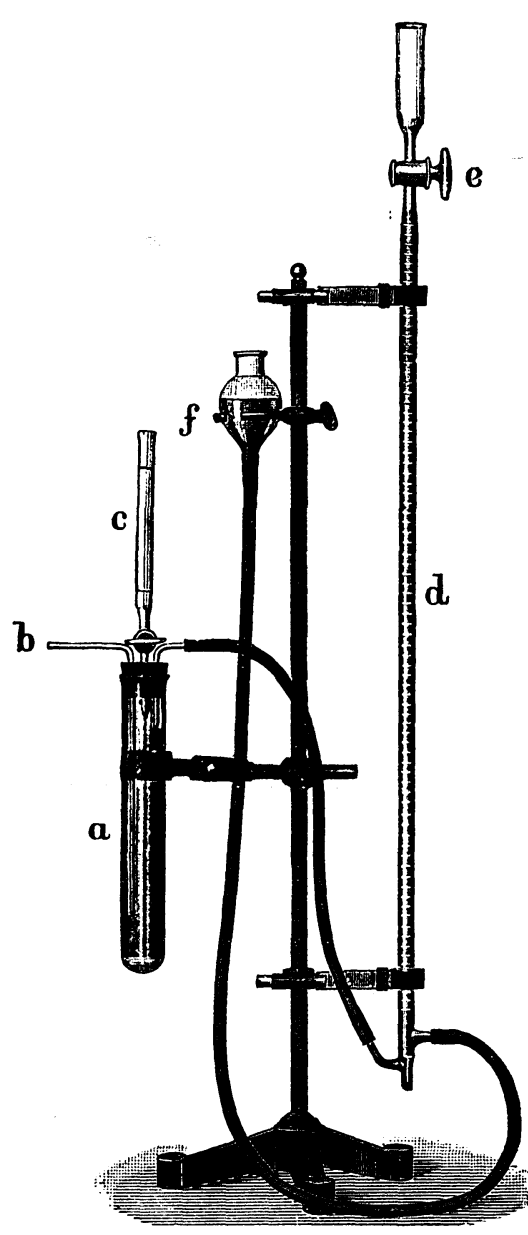

$3 \mathrm{~cm}$ von dem Boden des Gefäßes ab. Durch dies letztere wird zuerst mittels eines Stromes von Kohlensäure die Luft aus dem Apparat entfernt und nach Beendigung der Reaktion das gebildete Stickstoffgas in das Meßrohr übergeführt.

Beim Ausführen der Bestimmung verfährt man in folgender Weise: Mit Hilfe einer Pipette wird $1 \mathrm{ccm}$ sehr genau abgemessener Harn in das Gasentwicklungsgefäß $a$ gebracht und aus einem geeigneten Kohlensäureentwickler wird ein Strom von luftfreier Kohlensäure den Apparat durchgeleitet. Dabei muß der Hahn $e$ geöffnet sein und die Niveaukugel wird gesenkt, so daß das Meßrohr nur eine geringe Menge Kalilauge enthält. Nachdem der Berechnung gemäß der Apparat von Luft befreit ist, wird dieser durch Füllung des Meßrohres mit Kalilauge kontrolliert, worauf der Hahn $e$ geschlossen wird. ${ }^{1}$ ) Der Scheidetrichter wird danach bis zu der Marke 0 mit

1) Um das in dem Trichterrohr eingeschlossene Luftvolumen $\mathrm{zu}$ entfernen, wird auch dieses mit Kohlensäure gefüllt, wobei der Hahn während einiger Augenblicke geöffnet und die Verbindung mit dem Meßrohr geschlossen zu halten ist. 
dem von Riegler vorgeschriebenen Reagens ${ }^{1}$ ) gefüllt, und von diesem werden $2 \mathrm{ccm}$ durch Öffnen des Hahns in das Gasentwicklungsgefäß gebracht. Die Mischung von Harn mit Reagens wurde sodann mit Hilfe einer sehr kleinen Bunsenflamme einige Sekunden im Kochen gehalten, worauf der Hahn zu dem Kohlensäureentwickler wieder geöffnet wird. Durch einen schwachen Strom Kohlensäure wird der Stickstoff in das Meßrohr übergeführt, wobei die gleichzeitig übergehende Kohlensäure von der Kalilauge absorbiert wird. Sobald das Stickstoffvolumen in dem Meßrohr nicht mehr vermehrt wird, wird der Leitungsschlauch mittels eines Quetschhahns geschlossen und der Kohlensäurestrom abgesperrt. Nach fünf Minuten kann die Ablesung des Gasvolumens stattfinden, nachdem die Flüssigkeitsfläche in dem Meßrohr und die Niveaukugel in dieselbe Höhe gebracht worden sind, wobei man die Zimmertemperatur und den Barometerstand beobachtet. Die Prozentzahl des Harnstoffs wird aus der Formel

erhalten, wo

$$
\mathrm{p}=0,2141 \times \mathrm{v} \times \mathrm{g}
$$

$\mathrm{p}=$ Prozentzahl des Harnstoffs,

$\mathbf{v}=$ Stickstoffvolumen,

$\mathrm{g}=$ Gewicht von $1 \mathrm{ccm}$ Stickstoff in Milligrammen bei herrschender Temperatur und Druck.

$0,2141=$ konstanter Faktor. ${ }^{2}$ )

Bei einer Bestimmung hat man z. B. 10,7 ccm Stickstoff bei $16^{\circ}$ und $753 \mathrm{~mm}$ erhalten:

$$
p=0,2141 \times 10,7 \times 1,1495 ; p=2,63 \% .
$$

1) Millons Reagens etwas modifiziert.

$\left.{ }^{2}\right)$ Der Faktor 0,2141 wird aus den Gleichungen

erhalten.

$$
\left\{\begin{array}{l}
60,1004\left[\mathrm{CO}\left(\mathrm{NH}_{2}\right)_{2}\right]: 28,08\left[\mathrm{~N}_{2}\right]=\mathrm{x}:(\mathrm{v} \times \mathrm{g}) \\
1000: \mathrm{x}=100: \mathrm{p}
\end{array}\right.
$$

$$
\begin{gathered}
\mathrm{x}=\frac{60,1004 \times \mathrm{v} \times \mathrm{g}}{28,08} \\
\mathrm{p}=\frac{100 \mathrm{x}}{1000}=\frac{60,104 \times \mathrm{v} \times \mathrm{g}}{10 \times 28,08}=0,2141 \times \mathrm{v} \times \mathrm{g} \\
\mathrm{x}=\text { Gewicht in Milligrammen der dem Stickstoffvolumen } \mathrm{v} \\
\text { entsprechenden Menge Harnstoff. }
\end{gathered}
$$


176 Ekecrantz u. Söd erman, Bestimmung der Harnstoffmenge im Harב.

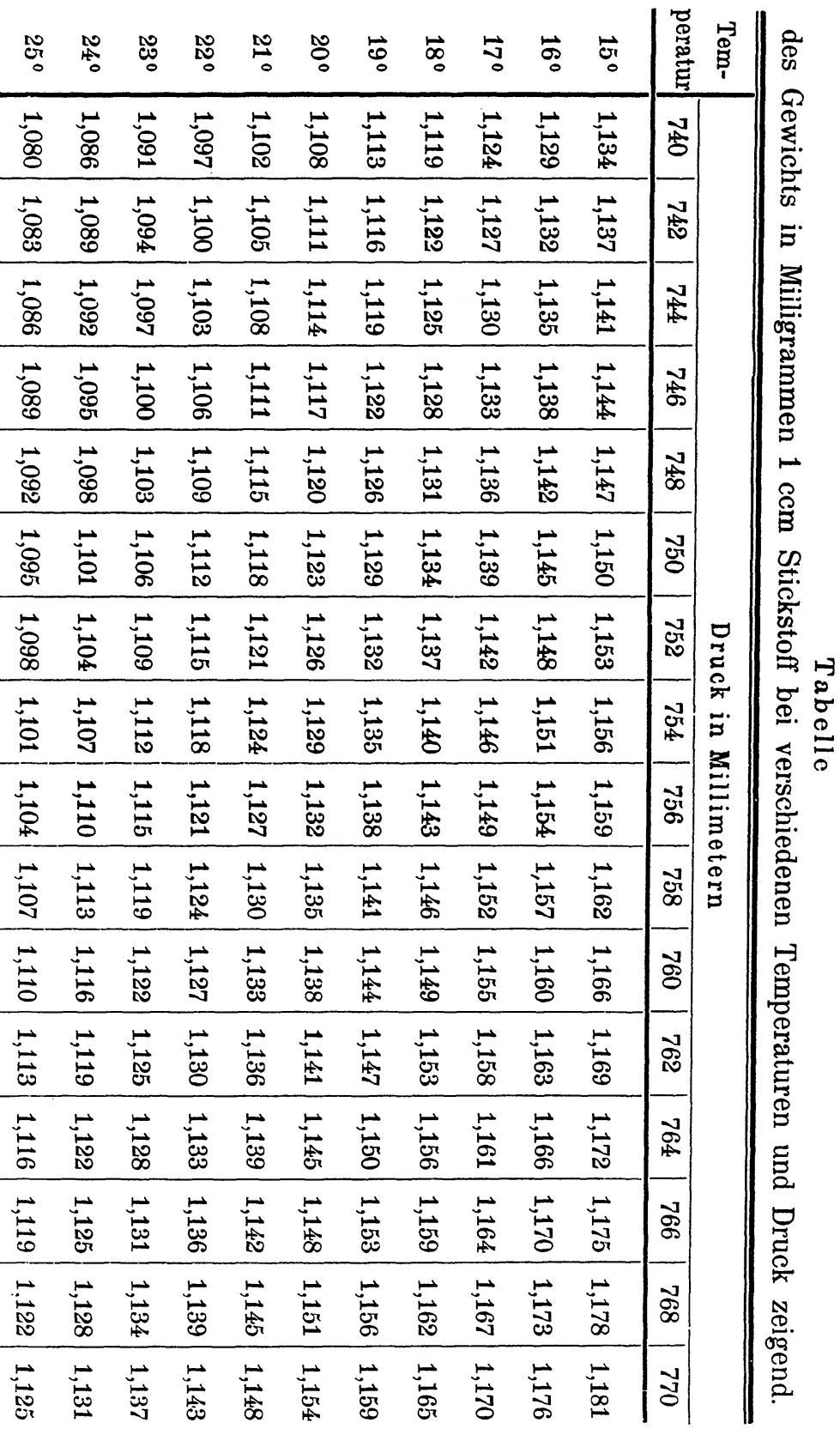

